\title{
Exploring User Input Metaphors for Jump and Run Games on Mobile Devices
}

\author{
Kolja Lubitz and Markus Krause \\ University of Bremen \\ \{phateon, pinguin\} atzi.de
}

\begin{abstract}
Mobile devices are already an important platform for digital games. These devices need specialized input metaphors as they have various restrictions such as their own hardware capabilities and the lack of external input devices. Especially challenging are fast paced interactions as in Jump and Run games. This paper explores three user inputs for Jump and Run games on mobile devices along the game Somyeol.
\end{abstract}

\section{Introduction}

Games for mobile devices are already common and use a range of input methods. The most common input device for games on mobile devices is their touchscreen. Examples can be found here (Parhi \& Karlson, 2006). Various games such as "Angry Birds" or "Cordy" use the touchscreen of mobile devices as the only input. The game "Angry Birds" for instance allows the player to shoot bird like characters with a simple sling metaphor. By dragging the sling around an anchor point the player chooses direction as well as strength of shot. Interaction in "Angry Birds" is reduced to this gesture. A classic Jump and Run game such as "Cordy" need more than one metaphor. The main challenge with a gamepad like input as this implemented for "Cordy" is that hitting the right movement button is difficult. In games like "Tiny Wings" or "Ninja Run" the problem is bypassed by letting the player run automatically.

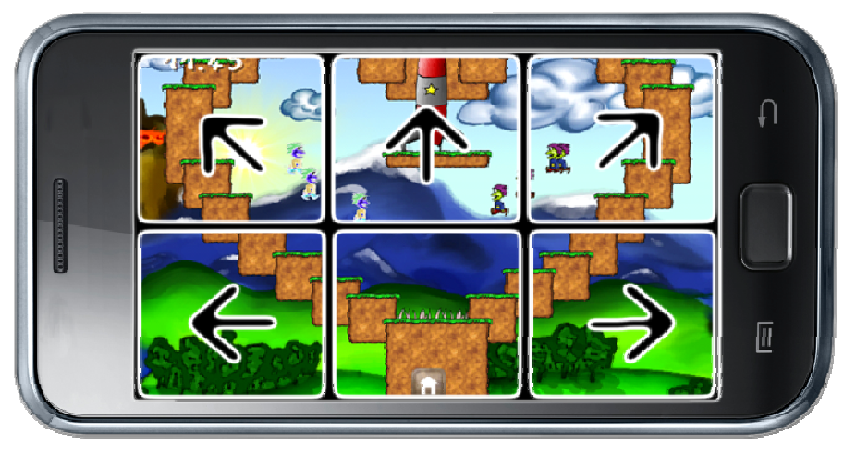

Fig. 1. A screenshot of the Somyeols game. The overlay illustrates the multi touch input 


\section{Input Methods}

This paper investigates three different user input methods for Jump and Run games on mobile devices or simply JRGMs. It illustrates its findings along the mobile JRGM Somyeol. Somyeol is a puzzle Jump and Run game in which the player controls more than one character at a time. The goal is to control as much Somyeols as possible through the levels. Somyeol is a game which needs input metaphors for three different actions: move left, move right, and jump. The three following input methods were implemented for Somyeol.

1. MultiTouch Input: The multi-touch method uses only the touch screen for input. The screen is split into six areas as depicted in Fig. 1. The grid maximizes the actual button size as the whole screen is active. The lower left and right areas are used for walking, top left and right areas for jumping in the respective direction. In the top center a straight jump up action is triggered. The bottom center does not have an associated action.

2. One-Finger Input: The One-Finger input method getting the first touch point from the screen. If the touch point moves left or right the player moves. If the touch point moves up the player jumps, if the finger moves up and left the player jumps left. This approach was inspirited by gamepad analog sticks.

3. Accelerometer Input: This input method uses the accelerometer of many mobiles phones to get the device tilt. Left and right tilt allows for movement in the respective direction. A touch gesture is used to trigger jumping. We also tried to trigger the jump if the device moving up, but this easily moves the device out of the players focus. So we decide to use the whole touch screen as a jump button. This approach is inspirited by car driving games and "Doodle Jump" where the device tilt is used to going right or left. Gilberston et. al gives examples on tilt as an input method (Gilbertson, Coulton, \& Chehimi, 2008).

\section{Evaluation}

The conducted study had 37 participants between 20 - 30 years old, 10 out of the 37 participants were women. Each participant played three test levels. Every level contains a certain challenge to test the input quality of the methods. After playing the participants answer a survey with 18 questions. These questions were integrated into the game and answered on the device the participant played with. The question whether the game was fun was answered on a 6-point Likert scale. The accelerometer input performed best with a mean of 4.27, multitouch (3.88) and one finger input (3.70) showed very similar results. The differences between the three input methods were however not significant. All $\mathrm{p}$ values measured with one tailed t-tests were $>0.15$. After publishing the game to a broader audience it turned out that accelerometer and multi-touch input were too demanding for players as both methods gained negative player feedback. This feedback was unexpected as questions in the survey about the usability of the input methods did not reveal any issues with any of the input methods. 


\section{References}

Gilbertson, P., Coulton, P., Chehimi, F.: Using Tilt as an Interface to Control No-Button 3-D Mobile Games. Computers in Entertainment (CIE) 6(3) (2008)

Parhi, P., Karlson, A.: Target size study for one-handed thumb use on small touchscreen devices. In: Proceedings of the 8th Conference on Human-Computer Interaction with Mobile Devices and Services, pp. 203-210 (2006) 